\title{
Optimizing Reservoir Performance by Automatic Allocation of Well Rates
}

\author{
ZAKIROV, Iskander S., Russian Academy of Sciences, Moscow, Russia \\ AANONSEN, Sigurd Ivar, Norsk Hydro Research Center, Bergen, Norway \\ ZAKIROV, Emest, S., Russian Academy of Sciences, Moscow, Russia \\ PALATNIK, Boris M., Geomatic A/S, Stavanger, Norway
}

Paper presented at the 5th European Conference on the Mathematics of Oil Recovery, Leoben, Austria, 3-5 Sept., 1996

\begin{abstract}
Optimal utilization of production facilities is an important factor in field development planning and production forecasting. The problem may for instance consist of finding an optimal allocation of available gas or water handling capacity to ensure maximum oil production. Normally, this optimization is done on a day-byday basis. However, a bad strategy at an early stage of production may be devastating for the late time production from the field. In this paper, a method for optimizing net present value of oil production with respect to the well production rates as functions of time over the whole life time of the field is presented. A fully implicit, 3-D black-oil simulator is used to obtain the forward solution. The optimization algorithm is based on the optimal control theory approach, which facilitates a fast calculation of the objective function gradient with respect to pressures and constraints on the oil, water and gas production rates at well and group level. Well bottom-hole pressures are used as control parameters.
\end{abstract}

\section{INTRODUCTION}

The search for an optimal strategy of field development is a challenging problem, and there is a large number of factors which should be taken into account. In this paper, the problem of finding an optimal allocation of available gas and water handling capacity between the individual wells producing against a common production platform is considered.

Several authors ${ }^{1-6}$ have made attempts to introduce various linearizations of reservoir performance descriptions and entered these data into the same variations of linear programming.

After nonlinear and dynamic programming methods appeared, it became possible to remove restrictions common for linear programming concerning objective function and constraint relationships. ${ }^{7-11}$ The class of solved problems remained practically the same, but the problem statement became more realistic and closer to real situations.

Pontryagin's maximum principle opened new ways and methods, called optimal control methods, solving problems in optimization. ${ }^{12,13}$ Simultaneously with the development of Pontryagin's maximum principle, several papers were devoted to gradient methods application to optimization and control problems. ${ }^{14-17}$

Recently, methods for taking into account uncertainty in the optimization of oil recovery have also been introduced. ${ }^{19}$

Optimal control theory methods have recently been developed, and therefore the field of their implementation is still not exhausted. One of the advantages of optimal control theory methods is that the computational effort involved in the calculation of the objective function gradient is independent of the number of parameters. Thus, these methods are especially well suited for problems involving a large number of unknowns and are used in this study.

The problem is formulated to maximize the net present value of oil production over a fixed life time of the field. The problem is solved by coupling an optimizer based on the conjugate gradient method to a fully implicit, 3D, three-phase, black-oil reservoir simulator where the set of constraints is imposed on well group production for oil and gas components. Well bottom hole pressures are chosen as control parameters, and the objective function is maximized by finding the optimal value of bottom hole pressures as functions of time for each well. The efficiency of the method is illustrated by two test case studies. 


\section{PROBLEM STATEMENT}

The forward problem (the simulator equations) may be written in matrix format as

$$
\mathbf{F}\left(\mathbf{x}^{n}, \mathbf{x}^{n-1}, \mathbf{u}^{n}\right)=\mathbf{0},
$$

where $\mathbf{x}^{n}$ is the solution and $\mathbf{u}^{n}$ is the vector of control parameters at time step $n$. The total number of control parameters (unknowns) equals the number of wells times the number of time steps.

Assume that $N$ wells are producing against the same platform. The production is subject to the following constraints on total production

$$
\begin{aligned}
& \sum_{i=1}^{N} q_{o i}=q_{o t} \leq q_{o \max } . \\
& \sum_{i=1}^{N} q_{g i}=q_{g t} \leq q_{g \max }, \\
& \sum_{i=1}^{N} q_{w i}=q_{w t} \leq q_{w \max } .
\end{aligned}
$$

In addition, there may be constraints on the production from each well

$$
\begin{aligned}
q_{o i} & \leq q_{o i \max }, \ldots \\
q_{g i} & \leq q_{g i \max }, \ldots \\
q_{w i} & \leq q_{w i \max }, \ldots \\
{\left[p_{w}\right]_{i} } & \geq\left[p_{w}\right]_{i \min } .
\end{aligned}
$$

In matrix format the set of constraints, Eqs. (2)-(8), represents a system of inequality constraints which may be written

$$
\boldsymbol{\nu}\left(\mathbf{x}^{n}, \mathbf{u}^{n}\right) \leq 0 \text {. }
$$

At a given time step of the forward problem solution, some of the inequality constraints, Eq. (9), will be active $\left(\nu_{j}=0\right)$ and some inactive $\left(\nu_{j}<0\right)$. Let the total number of active constraints be $k$ and the total number of unknowns be $l$ at a given time step. The number of free parameters is then $m=l-k$, and the vector of control parameters $\mathbf{u}^{n}$ may be splitted into the components $\mathbf{u}^{n}=\left(\mathbf{u}_{d}^{n}, \mathbf{u}_{f}^{n}\right)$, where $\mathbf{u}_{d}^{n}$ has dimension $k$ and represents the dependent control parameters, and $\mathbf{u}_{f}^{n}$ has dimension $m$ and represents the free control parameters.

In this study, discounted oil production is used as objective function, but the procedure may easily be extended to include also cost functions. The objective function depends on the total number of free parameters over the fields life time, and is defined by

$$
\begin{aligned}
J\left(\mathbf{u}_{f}\right) & =\sum_{n=1}^{n_{t}} f^{n}\left(\mathbf{x}^{n}, \mathbf{u}^{n}\right) \Delta t \\
& =\sum_{n=1}^{n_{t}} \frac{\bar{q}_{0}^{n} \Delta t}{(1+0.01 r)^{(n-1 / 2)}},
\end{aligned}
$$

where $\bar{q}_{o}^{n}$ is an average oil flow rate over time interval $\Delta t ; n_{t}$ is the total number of time intervals; and $r$ is discount rate over time interval $\Delta t$ in $\%$. In this study, monthly time intervals were used.

The optimal control problem consists of finding $\mathbf{u}_{f}^{\text {opt }}$ satisfying Eqs. (1) and (9) such that

$$
J\left(\mathbf{u}_{f}^{\text {opt }}\right) \geq J\left(\mathbf{u}_{f}\right), \quad \forall \mathbf{u}_{f} \in U .
$$

The set $U$ is determined by control constraints.

\section{SOLUTION TECHNIQUE}

To maximize objective function the conjugate gradient method is used. The estimate of the control vector $\mathbf{u}_{f}$ at the $\nu$-th iteration is obtained as a step of the gradient search procedure:

$$
\begin{aligned}
& \mathbf{u}_{f}^{\nu+1}=\mathbf{u}_{f}^{\nu}+\beta^{\nu} \mathbf{d}^{\nu} \ldots \\
& \mathrm{d}^{\nu}=\nabla J^{\nu}+\gamma^{\nu-1} \mathbf{d}^{\nu-1} \\
& \gamma^{\nu-1}=\frac{\left\|\nabla J^{\nu}\right\|^{2}}{\left\|\nabla J^{\nu-1}\right\|^{2}} .
\end{aligned}
$$

Here $\nabla J$ is the objective function gradient; $\mathbf{d}$ is a conjugate direction; and $\beta$ is a step size along the conjugate direction.

The method for evaluating the objective function gradient follows the procedure presented in Ref. 20 and is based on the assumption that the first variation of objective function is the part of functional increment that is linear in the variations of $\delta \mathbf{x}^{n}$ and $\delta \mathbf{u}^{n}$.

The expression for calculation of objective function gradient with respect to control parameters is then given by

$$
\begin{aligned}
\nabla \mathbf{J}= & \sum_{n=1}^{n_{i}}\left\{-\left[\frac{\partial \mathbf{F}^{n}}{\partial \mathbf{u}_{f}^{n}}\right]^{T} \Psi^{n}\right. \\
& \left.-\left[\frac{\partial \widetilde{\nu}^{n}}{\partial \mathbf{u}_{f}^{n}}\right]^{T} \boldsymbol{\Psi}^{n}+\frac{\partial f^{n}}{\partial \mathbf{u}_{f}^{n}}\right\} \Delta t,
\end{aligned}
$$

where $\widetilde{\boldsymbol{\nu}}$ represents the active components of Eq. (9). $\boldsymbol{\Psi}^{n}$ is the solution of the following adjoint system of equations

$$
\begin{aligned}
{\left[\frac{\partial \mathbf{F}^{n}}{\partial \mathbf{x}^{n}}\right]^{T} \boldsymbol{\Psi}^{n} } & =-\left[\frac{\partial \mathbf{F}^{(n+1)}}{\partial \mathbf{x}^{n}}\right]^{T} \boldsymbol{\Psi}^{(n+1)} \\
& -\left[\frac{\partial \widetilde{\nu}^{n}}{\partial \mathbf{x}^{n}}\right]^{T} \boldsymbol{\Psi}^{n}+\frac{\partial f^{n}}{\partial \mathbf{x}^{n}},
\end{aligned}
$$




$$
\begin{aligned}
{\left[\frac{\partial \mathbf{F}^{n}}{\partial \mathbf{u}_{d}^{n}}\right]^{T} \boldsymbol{\Psi}^{n}+\left[\frac{\partial \tilde{\boldsymbol{\nu}}^{n}}{\partial \mathbf{u}_{d}^{n}}\right]^{T} \boldsymbol{\Psi}^{n} } & =\frac{\partial f^{n}}{\partial \mathbf{u}_{d}^{n}} \\
n & =N, N-1, \ldots, 1,
\end{aligned}
$$

$$
\Psi^{(N+1)}=0 \text {. }
$$

The step size value along the search direction is determined as described in the following.

The dependent control parameters may be expressed in terms of the free control parameters, and it follows from Eq. (9) that each component of $\nu$ must satisfy:

$$
\nu_{j}\left(\mathbf{x}^{n}+\beta_{j} \delta \mathbf{x}^{n}, \mathbf{u}_{f}^{n}+\beta_{j} \mathbf{d}\right) \leq \mathbf{0}
$$

where $\delta \mathrm{x}^{n}$ is a solution of the problem for variations:

$$
\begin{aligned}
& \frac{\partial \mathbf{F}}{\partial \mathbf{x}^{n}} \delta \mathbf{x}^{n}+\frac{\partial \mathbf{F}}{\partial \mathbf{x}^{n-1}} \delta \mathbf{x}^{n-1} \\
& +\frac{\partial \mathbf{F}}{\partial \mathbf{u}_{d}^{n}} \delta \mathbf{u}_{d}^{n}+\frac{\partial \mathbf{F}}{\partial \mathbf{u}_{f}^{n}} \mathbf{d}=\mathbf{0} \\
& \frac{\partial \tilde{\boldsymbol{\nu}}}{\partial \mathbf{x}^{n}} \delta \mathbf{x}^{n}+\frac{\partial \tilde{\boldsymbol{\nu}}}{\partial \mathbf{u}_{d}^{n}} \delta \mathbf{u}_{d}^{n}+\frac{\partial \tilde{\boldsymbol{\nu}}}{\partial \mathbf{u}_{f}^{n}} \mathbf{d}=\mathbf{0} \\
& \delta \mathbf{x}^{n}(t=0)=0
\end{aligned}
$$

By expanding Eq. (19) to the first order, we obtain

$$
\nu_{j}\left(\mathbf{x}^{n}, \mathbf{u}_{f}^{n}\right)+\beta_{j} \frac{\partial \nu_{j}^{n}}{\partial \mathbf{x}^{n}} \delta \mathbf{x}^{n}+\beta_{j} \frac{\partial \nu_{j}^{n}}{\partial \mathbf{u}_{f}^{n}} \mathbf{d} \leq \mathbf{0} . \ldots
$$

I.e.,

$$
\beta_{j \max }=\frac{\nu_{j}\left(\mathbf{x}^{n}, \mathbf{u}_{f}^{n}\right)}{\frac{\partial \nu_{j}^{n}}{\partial \mathbf{u}_{f}^{n}} \mathbf{d}+\frac{\partial \nu_{j}^{n}}{\partial \mathbf{x}^{n}} \delta \mathbf{x}^{n}} . \ldots \ldots \ldots \ldots \ldots \ldots
$$

$\nu_{j}\left(\mathbf{x}^{n}, \mathbf{u}_{f}^{n}\right)$ is known from the previous iteration of the gradient search procedure.

It follows that the step size along the search direction must satisfy the following inequality:

$$
\beta \leq \min _{i}\left(\beta_{i \max }\right)
$$

One way of determining $\beta$ is to use the maximum allowable value according to Eq.(25). However, this will result in a very slow convergence, since only one additional constraint can be attained at each iteration. Since we know that one of the constraints will be active for all the time steps, it follows that the number of iterations will be at least equal to the number of time steps of the forward problem.

In order to increase convergence rate of the method, a slight violation of Eq. (9) was allowed for the first iterations. This was obtained by introducing two tuning parameters: One parameter specifying the relative degree of violation (admissible interval) which was allowed for the first iteration, and one parameter specifying the rate of which the admissible interval should decrease with the number of iterations.

Notice that the necessary terms for calculating the objective function gradient, Eq. (15), is given by two systems of linear equations having the same matrix as the Jacobi matrix of the simulator equations, and that the dimension of these systems is independent of the dimension of the parameter vector, $\mathbf{u}$. One iteration of the search procedure thus consist of first solving the forward problem, Eq. (1), as for a normal simulation. Then the adjoint equations, Eqs. (16)-(18), are solved backwards in time to obtain $\Psi^{n}$, and Eqs (20)-(22) forward in time to obtain $\delta \mathbf{x}^{n}$. Finally, the updated solution is obtained from Eqs. (12)-(15).

\section{METHOD TESTING}

Two test cases were studied in order to check the method efficiency. These cases differed by the number of producing wells.

The scheme of well location for the first test case is presented in Fig. 1. A synthetic reservoir model was splitted into two zones with different permeabilities $k_{1}$ and $k_{2}$ with $k_{1}=2 k_{2}$. Two producing wells $\mathrm{P} 1$ and P2 were located in high and low permeable zones respectively. A transmissibility multiplier (barrier) was introduced to investigate the effect of the communication between the two zones on the result. One injector, Il, was located in the gas cap and voidage replacement mode was specified. The field production was limited by the following constraints:

- $q_{o \max }=3015 \mathrm{Sm}^{3} /$ day corresponding to a $10 \%$ of STOOIP per year withdrawal;

- $q_{g \max }=1.5 \cdot q_{\text {omax }} \cdot G O R_{\text {init }}=6.65 \cdot 10^{5} \mathrm{Sm}^{3} /$ day.

Initial gas-oil ratio is $157 \mathrm{Sm}^{3} / \mathrm{Sm}^{3}$. Discount rate was set to $1 \%$ per month.

Two reference cases were simulated without applying any optimization: 1) Rates allocated between the wells according to the wells' PI; and 2) A maximum utilization of available gas handling capacity based on the following strategy: i) Produce the well with the lowest PI (i.e., P2) at maximum field rate, keeping P1 shut until maximum field gas rate is reached $\left(t=t_{1}\right)$. ii) Open P1 and produce both wells keeping total field gas and oil rates at the maximum levels until the production of P2 reaches zero $\left(t=t_{2}\right)$. iii) Produce P1 at constant gas rate. The well production history for these reference cases are shown in Figs. 2-5.

To test method validity, we started from a rather poor initial guess corresponding to $100 \mathrm{Sm}^{3} /$ day for both producing wells during whole life time of the reservoir development. Fig. 6 shows the value of the objective function as a function of the number of iterations. It should be noted that the computation time for each iteration of the optimal search procedure is about 1.7-1.8 times the time for one forward simulation run. Total number of unknown parameters are 100 corresponding to the number of wells times the number of time intervals.

The solution of the optimal problem is a distribution of bottom hole pressures (control parameters) versus time (Fig. 7). Corresponding oil flow rates, gas oil 
ratios, and water-oil ratios for the two wells are shown in Figs. 8-10.

A comparison between the optimal solution and the reference cases is shown in Figs. 11, 12. The values of objective function for the 3 cases are: Ref.Case 1: 27.31, Ref.Case 2: 27.62, Optimal Search Procedure: 28.49. It is clearly seen from Figs. 11 and 12 that the solution using the optimal search procedure is better than the rate allocation based on well PI's. Regarding Reference Case 2, the plateau production is longer, but the total oil production drops very quickly giving a lower objective function value than for the optimal solution.

Note that the well gas-oil-ratios are approximately equal at all times for the optimal solution, indicating that equal $G O R$ 's for all the wells is the best strategy for this type of flow. In general, the optimal strategy for obtaining a maximum field oil rate at a day-byday basis will be to keep the derivative of oil rate with respect to gas rate equal for all the wells ${ }^{18}$, but it is easily shown that if the sensitivity of well $G O R$ to the well oil rate is low, this criterion reduces to the equal-GOR criterion. The plots show the results with an impermeable barrier separating the two reservoir zones. However, opening up the barrier had no effect on these conclusions.

To increase the contribution of the plateau production in the objective function, the discount rate was increased by a factor 5 , but the optimal solution remained approximately the same. Thus, a strategy based on producing the wells sequentially yields the maximum plateau production, but then the field production drops very quickly, and if total oil production (even discounted) shortly after end of plateau is used as a measure, it will be more optimal to produce both wells simultaneously trying to obtain equal $G O R$ for both wells at all times. That is, trying to keep an equal gas-front movement throughout the reservoir.

An automatic allocation of well rates will only be of academic interest if it is limited to small cases with just a couple of wells. As mentioned, one of the advantages of optimal control theory methods is that the gradient calculation is independent of the number of unknowns, i.e., in this case the number of wells. However, the optimization problem will be more difficult to solve when the number of unknowns increase, and to investigate the applicability of the method to a more realistic case, the procedure was tested also on a second model with 10 wells.

The scheme of well locations for this case is shown in Fig. 13. As before, there are two zones with different degree of heterogeneity separated by an impermeable barrier. Permeability distributions are illustrated at the bottom of the figure. Five wells are located in each zone, two injecting wells are located in the gas cap under voidage replacement mode for the corresponding zone. All producing wells are gathered at the same group of wells and belong to the same facility. Similar set of group constraints were imposed as for the first test case: $q_{\text {omax }}=2710 \mathrm{Sm}^{3} / \mathrm{day}$, $q_{\text {max }}=6.34 \cdot 10^{5} \mathrm{Sm}^{3} /$ day. The fluids in place for both cases are close. However, to increase the well spacing, the reservoir was made 3 times wider along $x$ axis and the reservoir thickness divided by 2 , resulting in a lower plateau period.

Objective function versus the number of iterations are shown in Fig. 14. The jump in objective function after 15 iterations is due to restarting the conjugate gradient calculation (resetting the search directions) and is a well known way of increasing convergence rate of conjugate gradient methods (see e.g. Ref. 21). A reasonably good solution is obtained after $20-25$ iterations, showing that even if the number of wells were increased by a factor five, the number of simulations (and thus the CPU time) needed was increased only by a factor $2-3$.

Field oil and gas flow rates are presented in Fig. 15,16. Oil production rates for all the 10 wells are shown in Fig. 17. Gas-oil ratios are depicted in Fig. 18. The GOR-curves are not fully coinciding in this case, indicating that the absolute maximum is not reached. However, the objective function is much less sensitive to variations in rate allocation, than the well gas-oil ratios, and there is no reason to believe that a much better solution could be achieved by continuing the search process.

\section{CONCLUSIONS}

A new method for automatic allocation of well production rates over the field's life time was developed.

The method is coupled to a fully implicit, $3 \mathrm{D}$, threephase, black-oil reservoir simulator.

Through the use of an optimal control theory approach and an efficient search algorithm, optimal allocation of rates between a large number of wells can be found with a reasonable computational effort.

The convergence rate of the method was significantly increased by allowing a slight violation of the constraints for the first iterations of the search procedure.

Maximizing plateau production (plateau length) may not be optimal with respect to obtaining the best economy over the fields life time.

\section{NOMENCLATURE}

Scalars : Italic letters

Vectors : Lower case bold letters (column vector)

Matrices : Upper case bold letters

Upper

index $T$ : Transposed matrix or vector

d $=$ vector of conjugate search direction

$\mathbf{F} \quad=$ Defined by Eq. (1)

$\frac{\partial \mathbf{F}}{\partial \mathbf{x}_{j}^{n}} \quad=$ Jacobi matrix for forward problem 
$f \quad=$ Defined by Eq. (10)

$\frac{\partial f}{\partial x}=$ Gradient of $f$ with respect to $\mathrm{x}$

$J \quad=$ objective function

$\nabla J \quad=$ objective function gradient

$\mathbf{u} \quad=$ vector of control parameters

$\mathbf{u}_{f} \quad=$ vector of free control parameters

$\mathbf{u}_{d} \quad=$ vector of dependent control parameters

$\mathrm{x} \quad=$ vector of state variables

$\nu \quad=$ vector of constraints

$\tilde{\boldsymbol{\nu}} \quad=$ vector of active (equality) constraints

$q_{\alpha \max }=$ Group constraint values (gas, oil, water)

$q_{\alpha i \max }=$ Well constraint values (gas, oil, water)

$\beta \quad=$ step value along the search direction

$\boldsymbol{\Psi} \quad=$ vector of adjoint variables

\section{ACKNOWLEDGMENTS}

The authors thank Norsk Hydro a.s for permission to publish this paper.

\section{REFERENCES}

1. Charnes, A. and Cooper, W.W.: Management Models and Industrial Applications of Linear Programming, John Wiley \& Sons, New York City (1961) II.

2. Attra, H.D., Wise, H.B., and Black, W.M.: "Application of Optimizing Techniques for Studying Field Production Operations," JPT (Jan. 1961) 82-86; Trans., AIME, 222.

3. O'Dell, P.M., Steubing, N.W., and Gray, J.W.: "Optimization of Gas Field Operation," JPT (April 1973) 419-25.

4. See, B.A. and Horne, R.N.: "Optimal Reservoir Production Scheduling by Using Reservoir Simulation," SPEJ (October 1983) 717-26.

5. Lo, K.K. and Holden, C.W.: "Use of Well Management Schemes for Rate Forecasts," paper SPE 24071 presented at the 1992 West Regional Meeting, Bakersfield, California.

6. Lo, K.K., Starley, G.P. and Holden, C.W.: "Application of Linear Programming to Reservoir Development Evaluations," paper SPE 26637 presented at the 68th Annual Technical Conference and Exhibition, Houston, Texas, Oct. 3-6, 1993.

7. Hupper, J.D.: "Scheduling Gas Field Production for Maximum Profit," SPEJ (June 1974) 274-79, Trans., AIME, 257.

8. Stoisits, R.F., Batesole, E.C., Champion, J.H. and Park, D.H.: "Application of Non- linear Adaptive Modelling for Rigorous Representation of Production Facilities in Reservoir Simulation," paper SPE 24898 presented at the 67th Annual Technical Conference and Exhibition, Washington, DC, October 4-7, 1992.

9. Carroll, J.A. and Horne, R.N.: "Multivariate Optimization of Production Systems," JPT (July 1992) 782-789, 831, Trans., AIME, 293.

10. Lang, Z.X. and Horne, R.N.: "Optimal Production Scheduling Using Reservoir Simulators: A Comparison of Linear Programming and Dynamic Programming Techniques," paper SPE 12159 presented at the 58th Annual Technical Conference and Exhibition, San Francisco, Ca, Oct. 5-8, 1983.

11. Fang, W.Y. and Lo, K.K.: "A General Well Management Scheme for Reservoir Simulation," paper SPE 29124 presented at the 13th Symposium on Reservoir Simulation, San Antonio, February 12-15, 1995.

12. Ramirez, W.F.: "Application of Optimal Control Theory to Enhance Oil Recovery," Developments in Petroleum Science, 21, Elsevier, Amsterdam, 1987.

13. Rowan, G. and Warren, J.E.: "A Systems Approach to Reservoir Engineering, Optimum Development Planning," J. Can. Pet. Tech. (July 1967) 84-94.
14. Ramirez, W.F., Fathi, Z. and Cagnol, J.L.: "Optimal Injection Policies for Enhanced Oil Recovery: Part 1 - Theory and Computational Strategies," SPEJ (June 1984) 328-32.

15. Fathi, Z. and Ramirez, W.F.: "Optimal Injection Policies for Enhanced Oil Recovery: Part 2 - Surfactant Flooding," SPEJ (June 1984) 333-41.

16. Vignes, O.: "Application of Optimization Methods in Oil Recovery Problems," Ph.D. Thesis, The Norwegian Institute of Technology, 1993.

17. Virnovsky, G.A. and Kleppe, H.: "Application of Control Theory to Optimize Horizontal Well Location Producing from a Thin Oil Zone," paper presented at the 8th European IOR-Symposium, Vienna, Austria, May 15-17, 1995.

18. Urbanczyk, C.H. and Wattenberger, R.A.: "Optimization of Well Rates Under Gas Coning Conditions," paper SPE 21677 presented at the Production Operations Symposium, Oklahoma City, Oklahoma, April 7-9, 1991.

19. Aanonsen, S.I., Eide, A.L. and Aasen, J.0.: "Optimizing Reservoir Performance Under Uncertainty with Application to Well Location," paper SPE 30710 presented at the SPE Annual Technical Conference and Exhibition, Dallas, October 22-25, 1995 .

20. Palatnik B.M., Aanonsen, S.I., Zakirov, I.S. and Zakirov, E.S.: "New Technique to Improve the Efficiency of History Matching of Full-Field Models," Proc., 4th European Conference on the Mathematics of Oil Recovery, Røros, Norway, June 7-10, 1994.

21. Fletcher, R.: Practical Methods of Optimization, John Wiley \& Sons, New York City (1987) Second Edition.

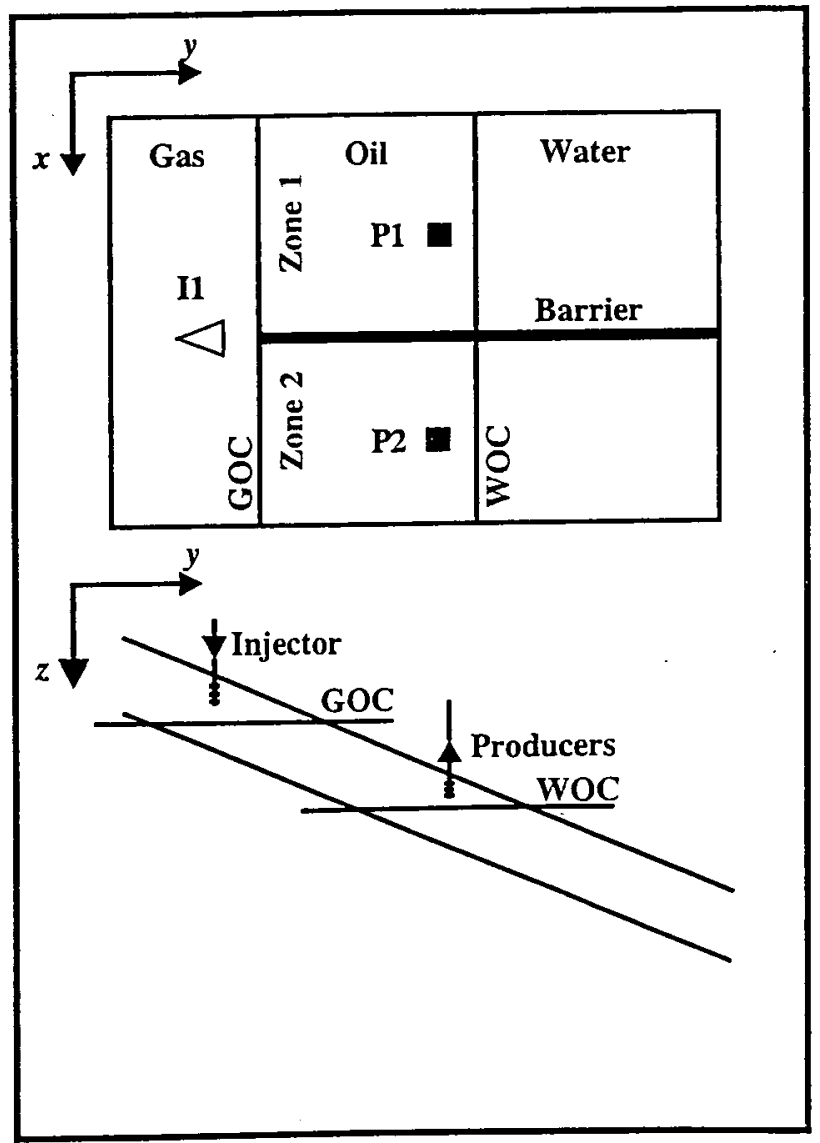

Fig. 1. 2-well model 


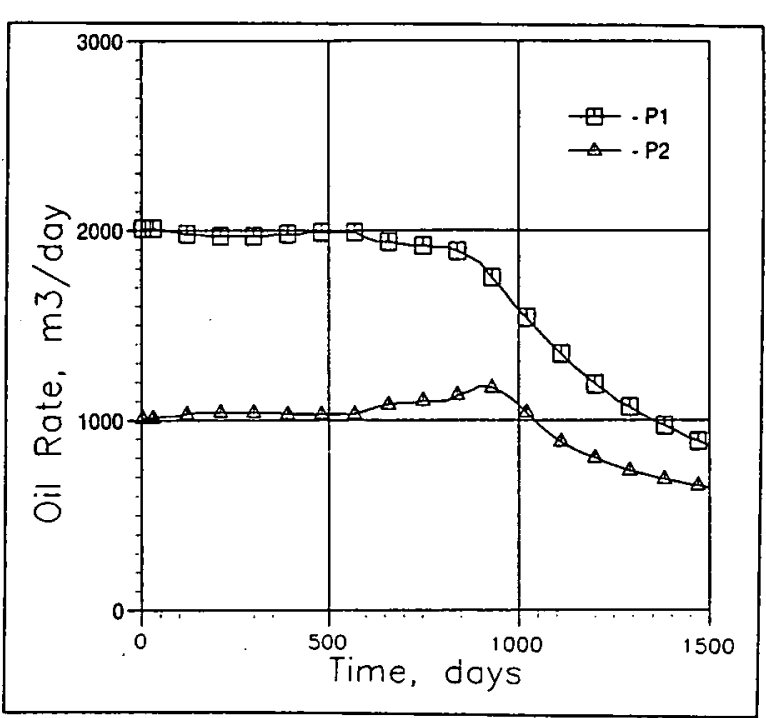

Fig. 2. Oil production rates reference case 1.

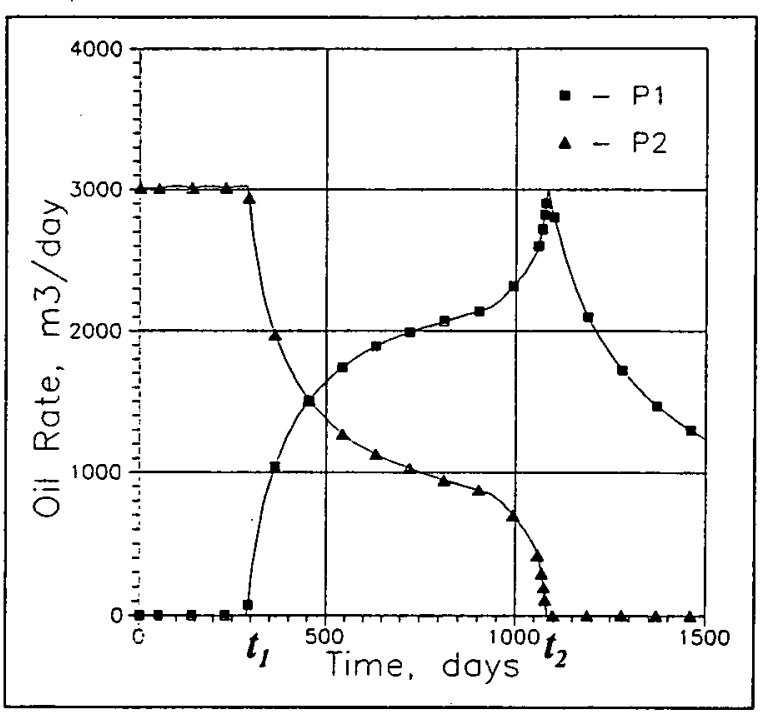

Fig. 4. Oil production rates reference case 2 .

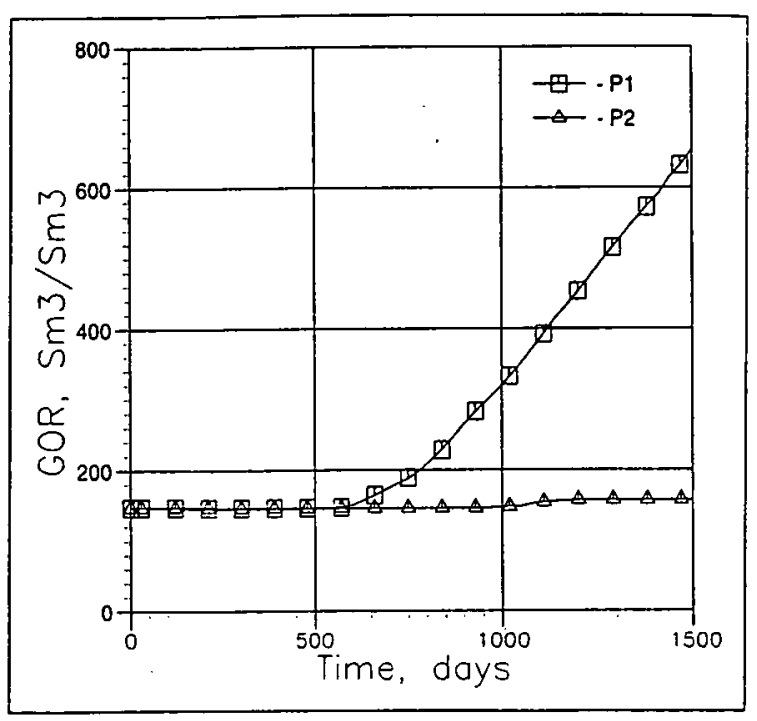

Fig. 3. Well gas-oil ratios reference case 1.

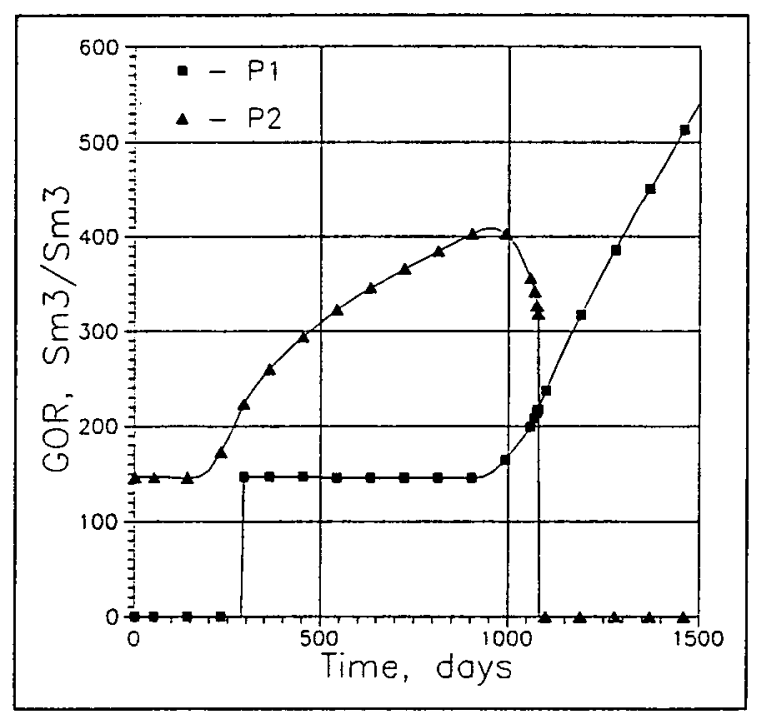

Fig. 5. Well gas-oil ratios reference case 2 .

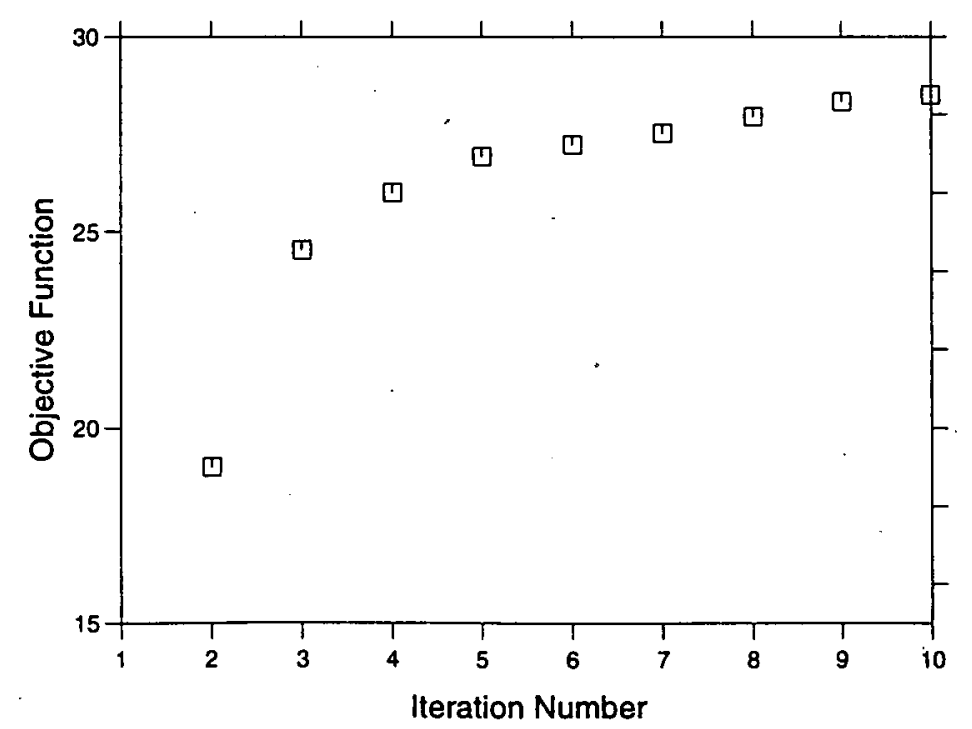

Fig. 6. Objective function vs. the number of iterations for the 2-well case. 


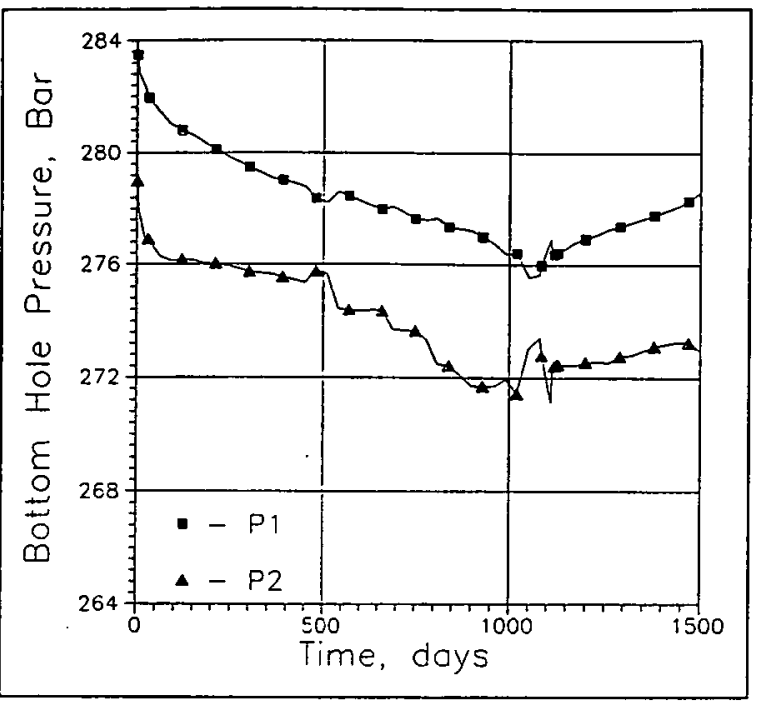

Fig. 7. Bottom-hole pressures for the optimal search procedure.

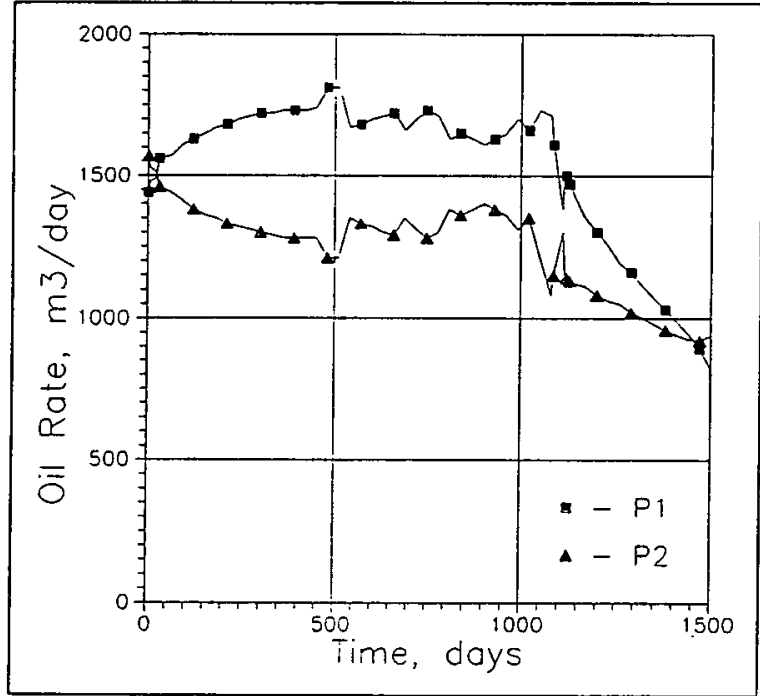

Fig. 9. Oil flow rates for the optimal search procedure.

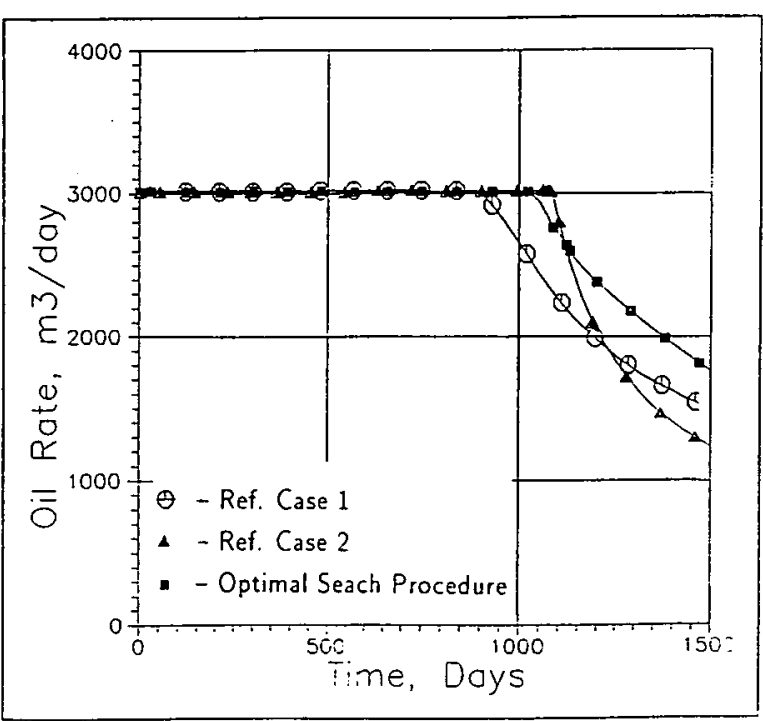

Fig. 11. Field oil production rates for the reference cases and optimal search procedure.

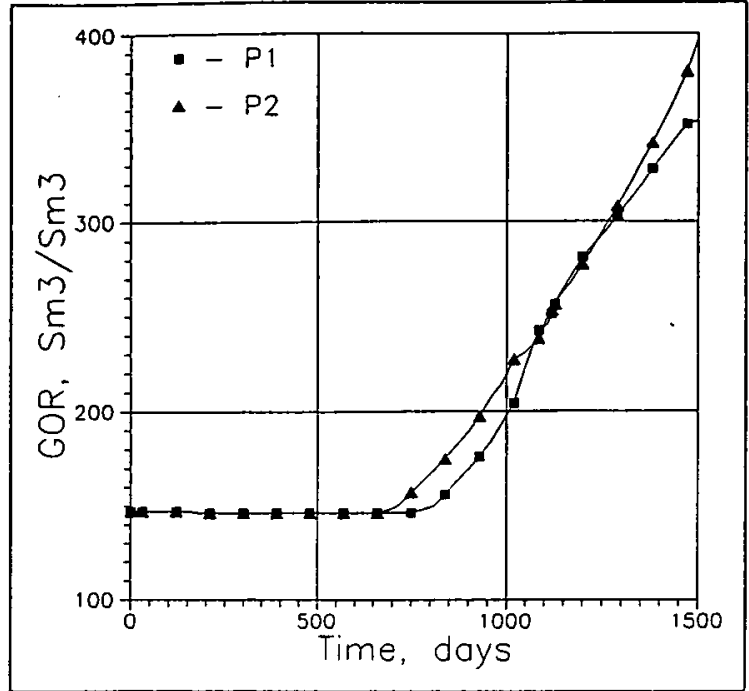

Fig. 8. Gas-oil ratios for the optimal search procedure.

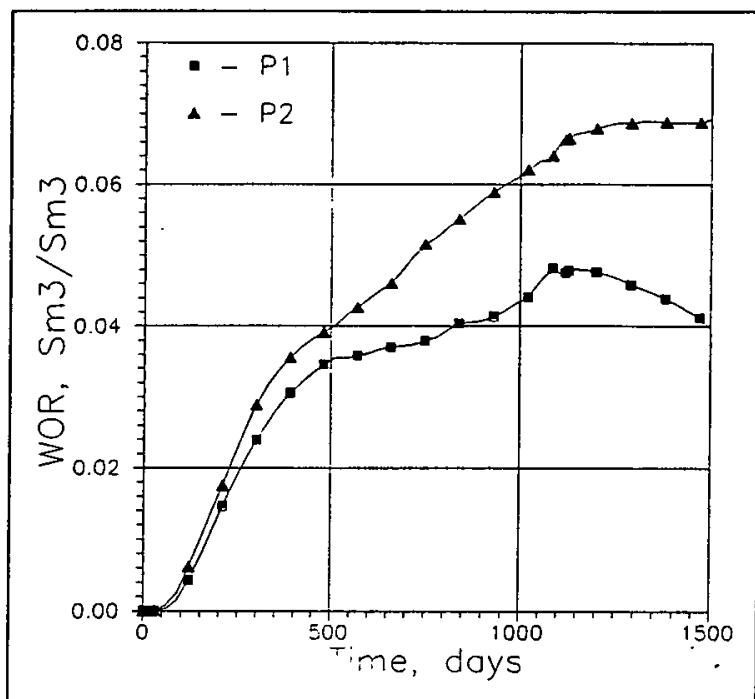

Fig. 10. Water-oil ratios for the optimal search procedure.

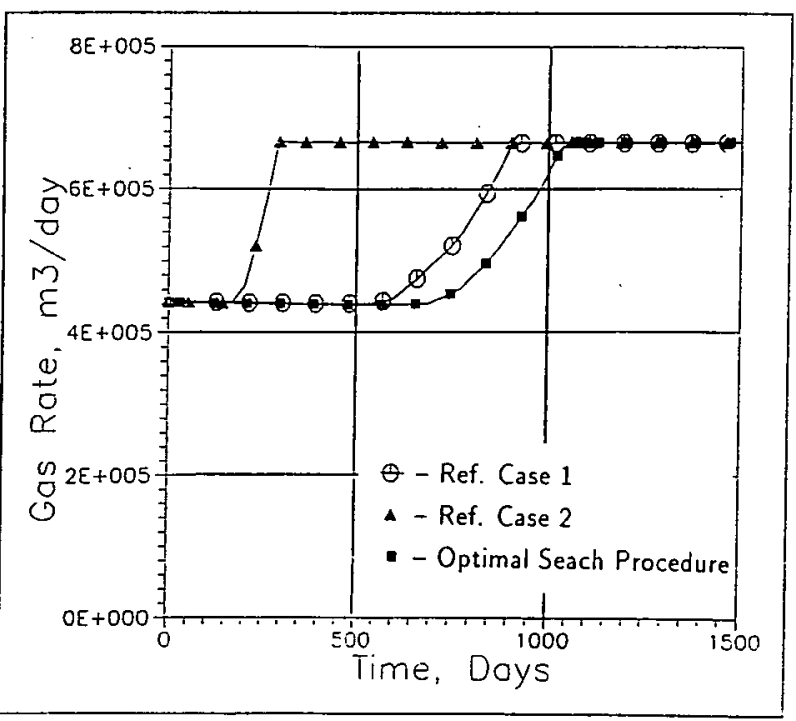

Fig. 12. Field gas production rates for the reference cases and optimal search procedure. 

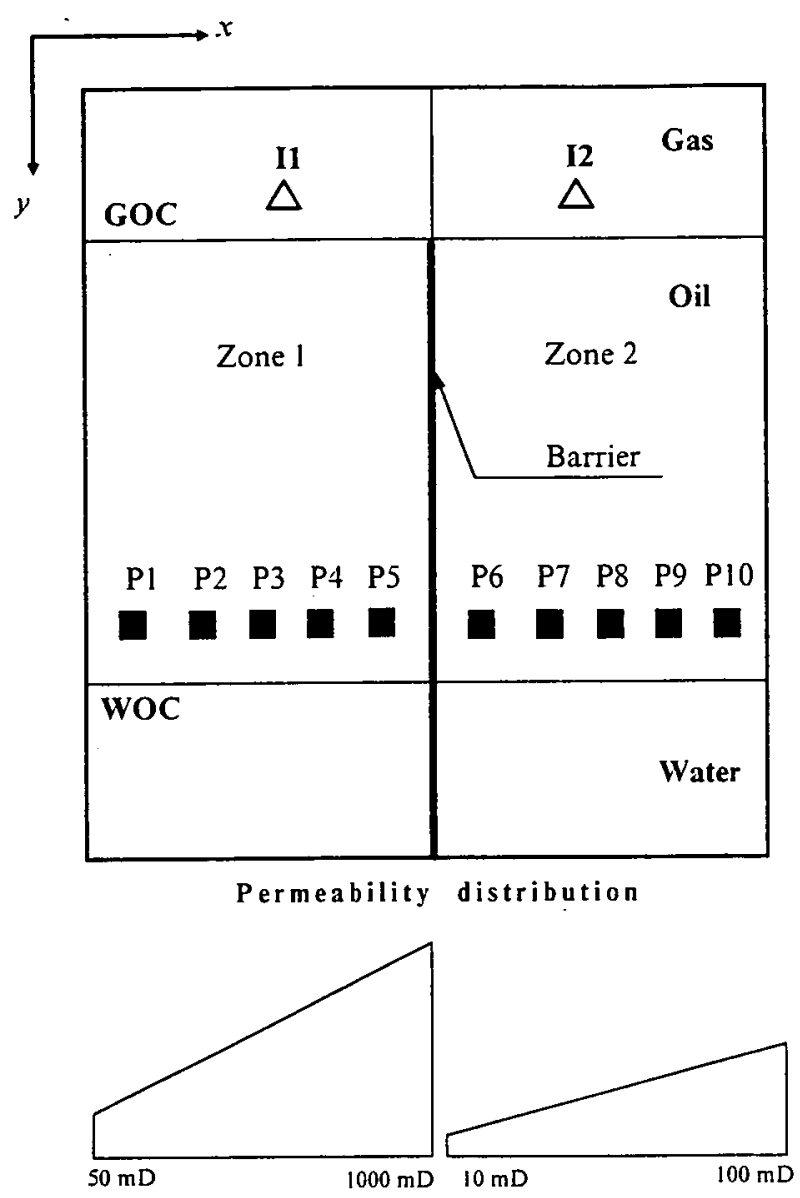

Fig. 13. 10-well model

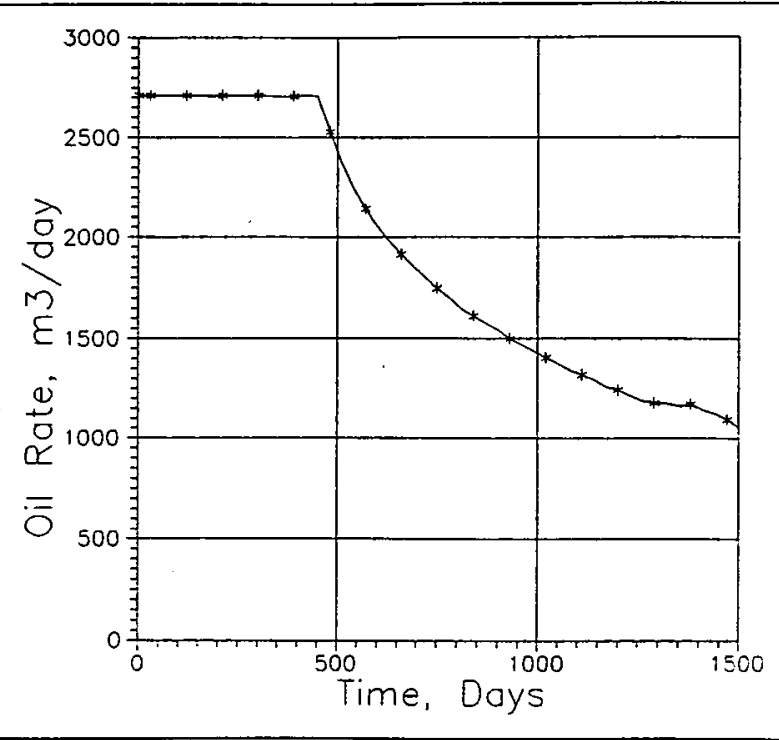

Fig. 15. Field oil rate 10 well case.

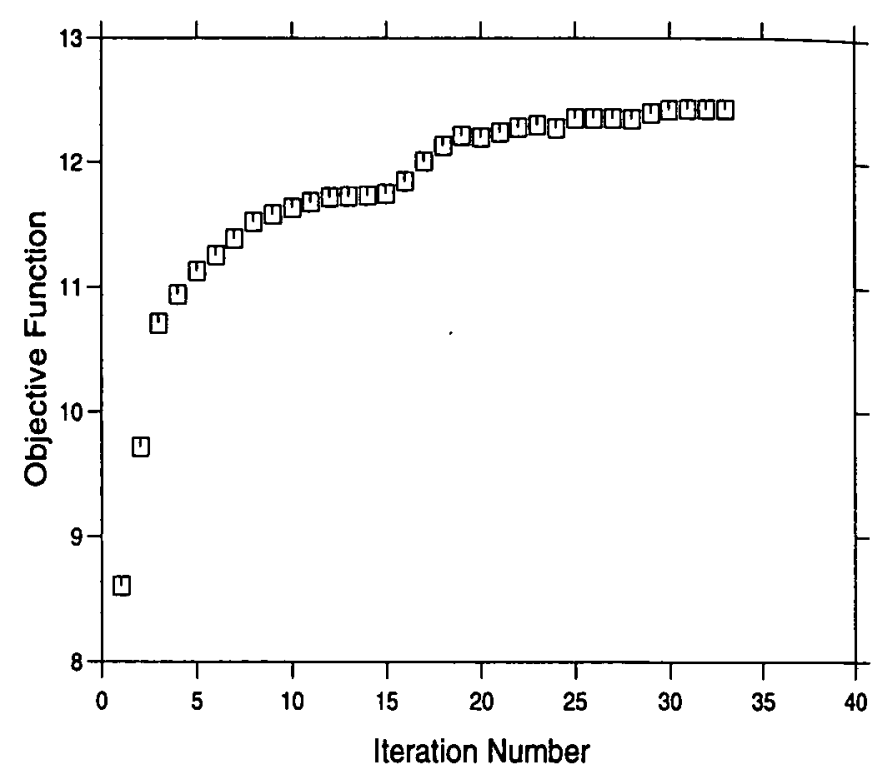

Fig. 14. Objective function vs. the number of iterations for the 10 -well case.

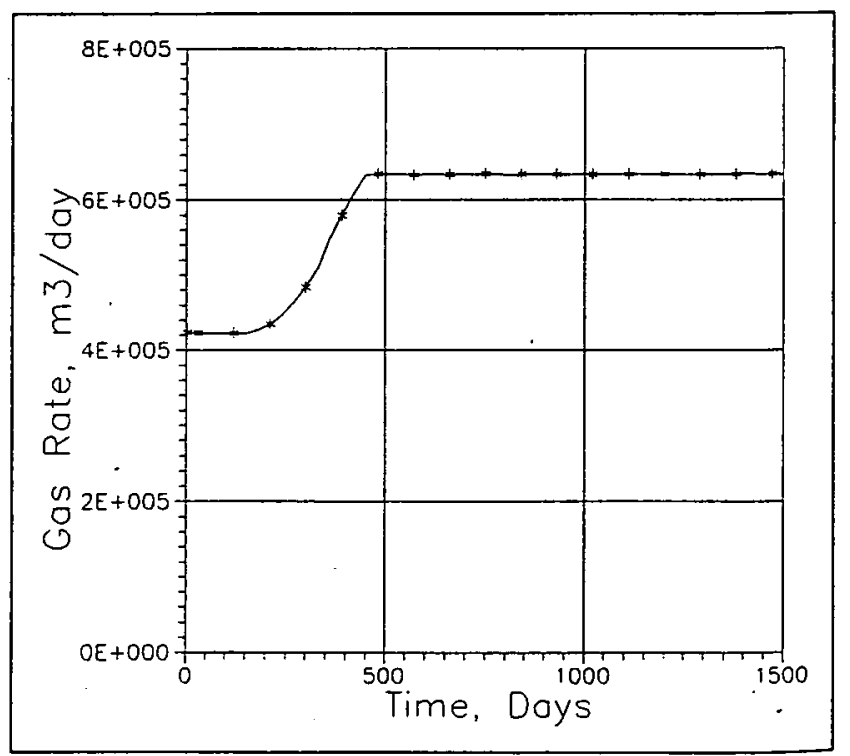

Fig. 16. Field gas rate 10 well case. 

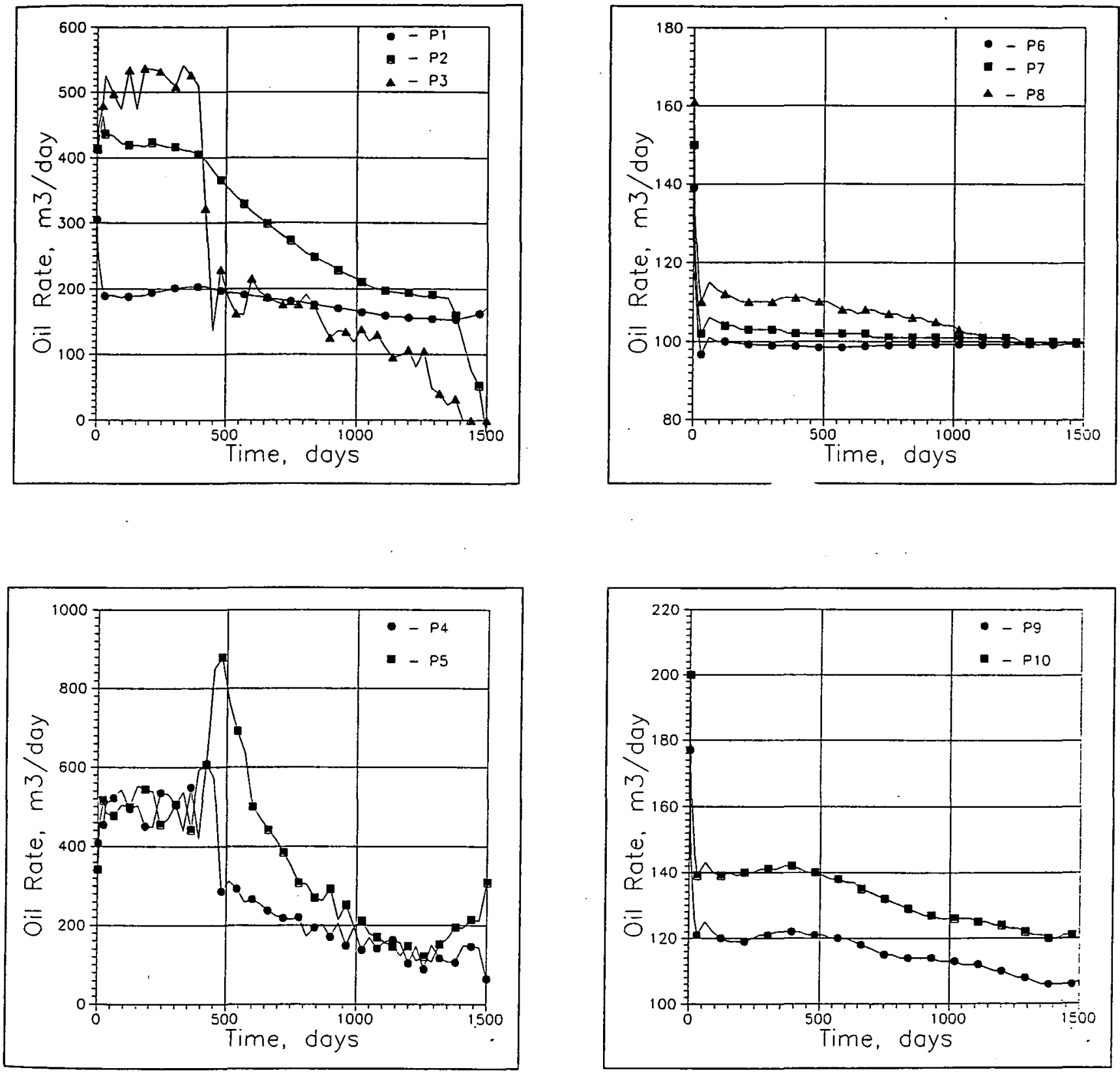

Fig. 17 Well oil production rates 10 -well case. 

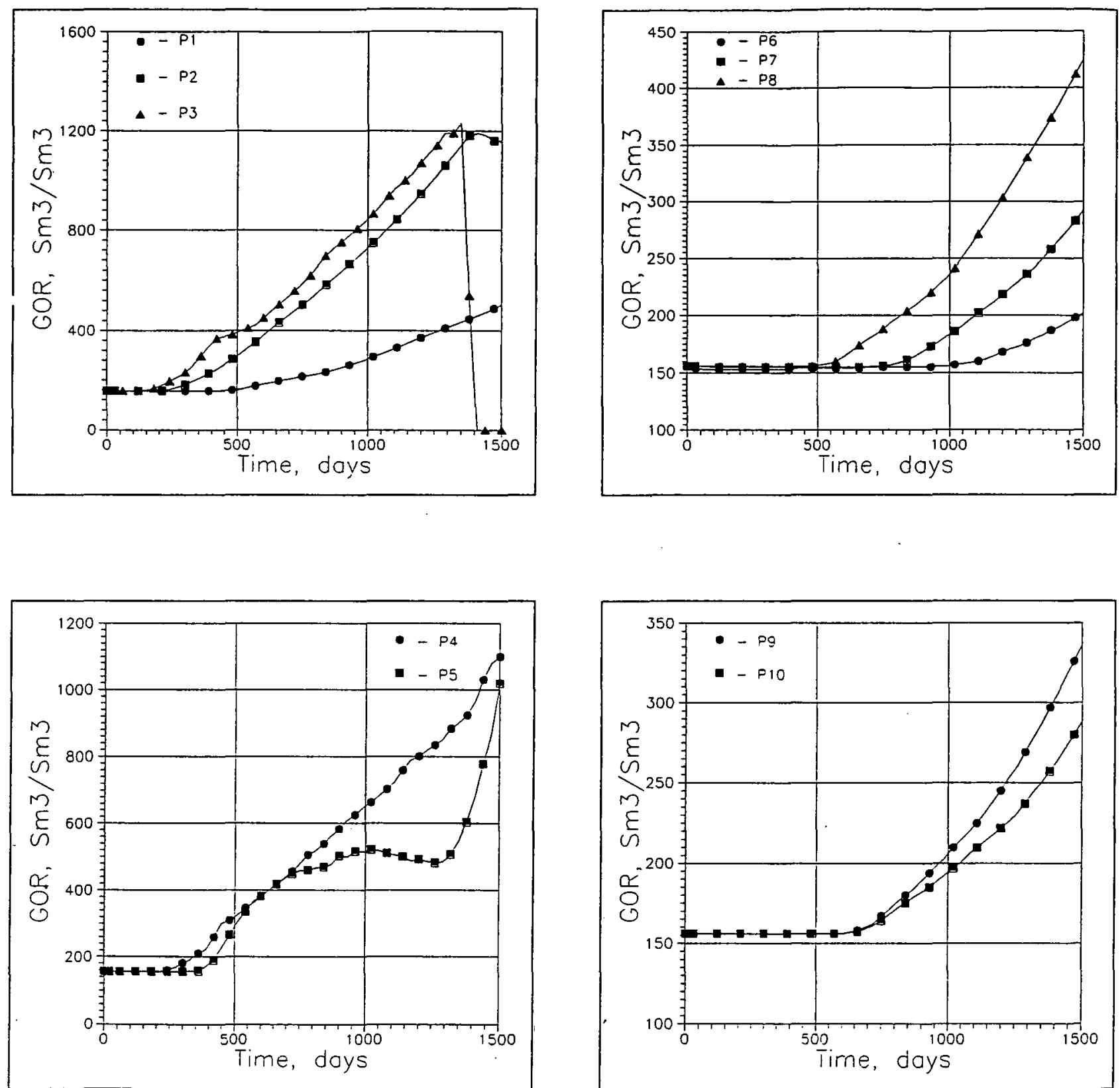

Fig. 18 Well gas-oil ratios 10-well case. 